\title{
GENERALIZED TELEGRAPH PROCESS WITH RANDOM DELAYS
}

\author{
DAOUD BSHOUTY, ${ }^{*}$ Technion - Israel Institute of Technology \\ ANTONIO DI CRESCENZO ***** AND \\ BARBARA MARTINUCCI, ${ }^{* * * * * *}$ Università di Salerno \\ SHELEMYAHU ZACKS, ${ }^{* * * * *}$ Binghamton University
}

\begin{abstract}
In this paper we study the distribution of the location, at time $t$, of a particle moving $U$ time units upwards, $V$ time units downwards, and $W$ time units of no movement (idle). These are repeated cyclically, according to independent alternating renewals. The distributions of $U, V$, and $W$ are absolutely continuous. The velocities are $v=+1$ upwards, $v=-1$ downwards, and $v=0$ during idle periods. Let $Y^{+}(t), Y^{-}(t)$, and $Y^{0}(t)$ denote the total time in $(0, t)$ of movements upwards, downwards, and no movements, respectively. The exact distribution of $Y^{+}(t)$ is derived. We also obtain the probability law of $X(t)=Y^{+}(t)-Y^{-}(t)$, which describes the particle's location at time $t$. Explicit formulae are derived for the cases of exponential distributions with equal rates, with different rates, and with linear rates (leading to damped processes).
\end{abstract}

Keywords: Telegraph process; delayed telegraph; alternating renewal; exponential distribution; damped process; compound Poisson process; hypergeometric series

2010 Mathematics Subject Classification: Primary 60G55

Secondary 60K20; 60G40

\section{Introduction}

Integrated telegraph processes have been studied by many authors. In particular, see Orsingher [15], Di Crescenzo [4], Di Crescenzo and Pellerey [7], and others. Zacks [19] studied a generalized process in which the times of motion up or down followed a general alternating renewal process. Stadje and Zacks [18] studied an integrated telegraph process in which at every turn, the particle followed different velocities chosen at random from a set of possible velocities.

In the present paper, denoting by $V(t), t \geq 0$, the velocity of a particle at time $t$, we consider a telegraph process such that either $V(t)=+1$ (up movement), $V(t)=-1$ (down movement), or $V(t)=0$ (no movement). More precisely, we consider a stochastic process in which $V(0)=1$ and the velocity does not change for a random length of time $U_{1}$. At $U_{1}$, we

Received 5 July 2011; revision received 15 December 2011.

Dedicated to Marcel Neuts on the occasion of his 75th birthday, in admiration of his most profound contributions to research and applications of stochastic processes.

* Postal address: Department of Mathematics, Technion - Israel Institute of Technology, Haifa 32000, Israel.

Email address: daoud@tx.technion.ac.il

** Postal address: Dipartimento di Matematica, Università di Salerno, I-84084 Fisciano (SA), Italy.

*** Email address: adicrescenzo@unisa.it

**** Email address: bmartinucci@unisa.it

***** Postal address: Department of Mathematical Sciences, Binghamton University, Binghamton, NY 13902-6000, USA. Email address: shelly@math.binghamton.edu 


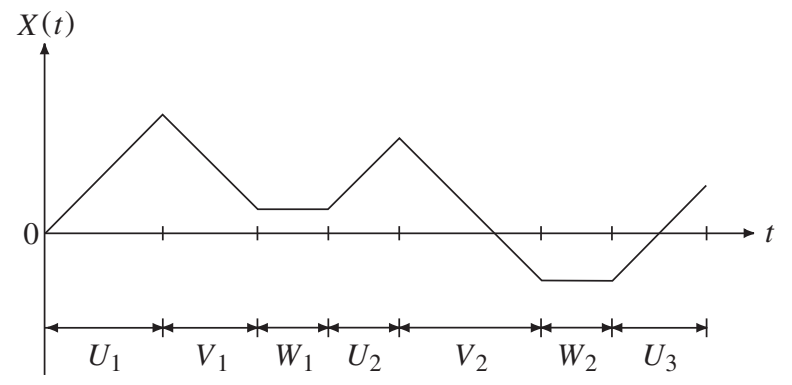

FIgURE 1: A sample path of $X(t)$.

have $V\left(U_{1}\right)=-1$ until the random time $U_{1}+V_{1}$. Then we have $V\left(U_{1}+V_{1}\right)=0$ until the random time $U_{1}+V_{1}+W_{1}$. This process repeats itself cyclically, following alternating renewals of $\left\{\left(U_{i}, V_{i}, W_{i}\right) ; i=1,2, \ldots\right\}$. The $i$ th renewal cycle is of length $U_{i}+V_{i}+W_{i}$. A sample path of $X(t)$ is provided in Figure 1. Here $\left\{U_{1}, U_{2}, \ldots\right\}$ are independent and identically distributed (i.i.d.) random times of movements upwards, $\left\{V_{1}, V_{2}, \ldots\right\}$ are i.i.d. random times of movements downwards, and $\left\{W_{1}, W_{2}, \ldots\right\}$ are i.i.d. random times of no-movement delays. Thus, the position of the particle after $n$ such cycles is

$$
X\left(\sum_{i=1}^{n}\left(U_{i}+V_{i}+W_{i}\right)\right)=\sum_{i=1}^{n}\left(U_{i}-V_{i}\right),
$$

where $X(t), t \geq 0$, denotes the position of the process at time $t$. More precisely,

$$
X(t)=\int_{0}^{t} \mathbf{1}_{\{V(s)=1\}} \mathrm{d} s-\int_{0}^{t} \mathbf{1}_{\{V(s)=-1\}} \mathrm{d} s, \quad t \geq 0 .
$$

Let us now introduce the total sojourn times in $(0, t)$. We thus set

$$
\begin{array}{rlrl}
Y^{+}(t) & =\int_{0}^{t} \mathbf{1}_{\{V(s)=1\}} \mathrm{d} s, & t \geq 0, \\
Y^{0}(t)=\int_{0}^{t} \mathbf{1}_{\{V(s)=0\}} \mathrm{d} s, & t \geq 0,
\end{array}
$$

and

$$
Y^{-}(t)=\int_{0}^{t} \mathbf{1}_{\{V(s)=-1\}} \mathrm{d} s, \quad t \geq 0 .
$$

Obviously, for all $t \geq 0$, the following identities hold:

$$
Y^{+}(t)+Y^{0}(t)+Y^{-}(t)=t, \quad X(t)=Y^{+}(t)-Y^{-}(t) .
$$

In the following section we develop the marginal distribution of the ancillary process $Y^{+}(t)$. This will yield, in Section 3, the distribution of $X(t)$. Finally, Section 4 is devoted to the analysis of various special cases obtained by specifying the probability laws of the random times $U_{i}, V_{i}, W_{i}$. The instances of exponential distributions with equal rates, with different rates, and with linear increasing rates (leading to damped processes) are investigated in detail.

It should be mentioned that random motions characterized by three cyclically alternating velocities have attracted the attention of various researchers. Indeed, Orsingher [16] provided 
a thorough investigation of the equations governing the probability law of a three-valued telegraph process. Di Matteo and Orsingher [9] extended Orsingher's investigation to the conditional distributions and the means of the process. More recently, Leorato et al. [14] studied the distributions of a one-dimensional motion with stops, viewed as the marginal of a two-dimensional motion with four cyclic velocities, whereas Leorato and Orsingher [13] considered a planar continuous-time random walk that moves with constant velocity along three possible directions. A planar random motion with three directions has also been studied by Cesarano and Di Crescenzo [3] and Di Crescenzo [5]. The more general case of cyclic random motions in $\mathbb{R}^{d}$ with $n$ directions has been considered by Lachal [12]. In the above papers an approach based mainly on analytic methods or on order statistics is employed, whereas the present contribution is grounded on a renewal theory basis.

\section{The distribution of $Y^{+}(t)$}

Let $U_{i}, V_{i}$, and $W_{i}(i=1,2, \ldots)$ have absolutely continuous distributions $F_{U}, F_{V}$, and $F_{W}$, respectively, and denote by $f_{U}, f_{V}$, and $f_{W}$ their corresponding densities. Let us set

$$
T_{i}=V_{i}+W_{i}, \quad i=1,2, \ldots,
$$

so that the distribution of $T_{i}$ is $F_{T}=F_{V} * F_{W}$, where, as usual, '*' denotes the convolution operator. Hence,

$$
F_{T}(t)=\int_{0}^{t} f_{V}(x) F_{W}(t-x) \mathrm{d} x, \quad t \geq 0 .
$$

Let $S_{0}^{U}=0$ and $S_{n}^{U}=\sum_{i=1}^{n} U_{i}, n=1,2, \ldots$, and define

$$
N_{U}(\omega)=\max \left\{n \geq 0: S_{n}^{U} \leq \omega\right\}, \quad \omega \geq 0 .
$$

We construct a new compound renewal process, namely,

$$
T_{U}(\omega)=\sum_{n=0}^{N_{U}(\omega)} T_{n}, \quad \omega>0
$$

with $T_{0}=0$. Hence, if $T_{U}(\omega)=t-\omega, 0<\omega \leq t$, this states that during $(0, t)$ the particle moves up for $\omega$ time instants and moves down or stays in place for $t-\omega$ time instants. Let us now note that process (1.1) identifies with the stopping time

$$
Y^{+}(t)=\inf \left\{\omega>0: T_{U}(\omega) \geq t-\omega\right\} .
$$

Note also that $T_{U}\left(Y^{+}(t)\right) \geq t-Y^{+}(t)$ for $t>0$, where $T_{U}\left(Y^{+}(t)\right)$ is the total time in $(0, t)$ that the particle moves down or stays in place, i.e. $T_{U}\left(Y^{+}(t)\right)=Y^{-}(t)+Y^{0}(t)$. In particular, for a fixed time $t>0$, we have

(i) $T_{U}\left(Y^{+}(t)\right)=t-Y^{+}(t)$ when the particle is moving upwards at time $t$,

(ii) $T_{U}\left(Y^{+}(t)\right)>t-Y^{+}(t)$ when the particle is either moving downwards at time $t$ or staying in place at time $t$.

Figure 2 shows $Y^{+}(t)$ for an instance in which case (ii) holds, where $S_{n}^{U}=\sum_{i=1}^{n} U_{i}$.

We remark that the cumulative distribution function (CDF) of $T_{U}(\omega)$ can be expressed as

$$
F_{T_{U}}(y ; \omega):=\mathrm{P}\left[T_{U}(\omega) \leq y\right]=\sum_{n=0}^{+\infty} p_{n}(\omega) F_{T}^{(n)}(y),
$$




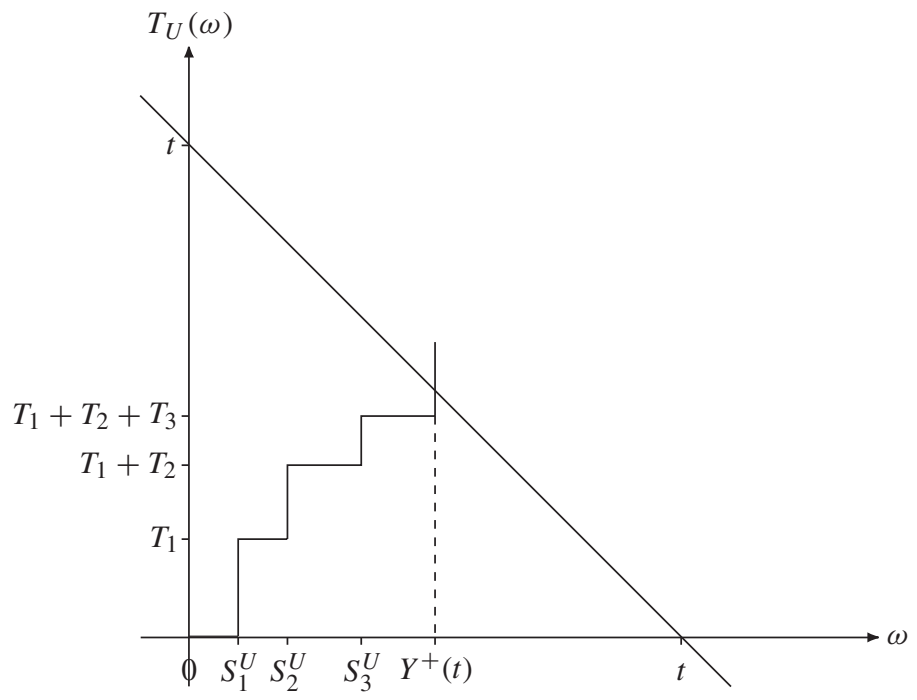

FIGURE 2: Sample paths of $T_{U}(\omega)$ and $Y^{+}(t)$.

where

$$
p_{n}(\omega):=\mathrm{P}\left[N_{U}(\omega)=n\right]=F_{U}^{(n)}(\omega)-F_{U}^{(n+1)}(\omega), \quad \omega>0, n \geq 0,
$$

and $\phi^{(n)}$ denotes the $n$-fold convolution of any function $\phi$, with $\phi^{(0)}=1$. Note also that $T_{U}(\omega)$ is a nondecreasing process. Therefore, making use of (2.5) and (2.6), the following result is straightforward.

Theorem 2.1. The probability law of $Y^{+}(t)$ is given by

$$
\mathrm{P}\left[Y^{+}(t)>y\right]=F_{T_{U}}(t-y ; y)=\sum_{n=0}^{+\infty} p_{n}(y) F_{T}^{(n)}(t-y), \quad 0<y<t,
$$

and

$$
\mathrm{P}\left[Y^{+}(t)=t\right]=1-F_{U}(t), \quad t>0 .
$$

From Theorem 2.1, hereafter we immediately obtain the density of the absolutely continuous component of $Y^{+}(t)$, denoted as $\psi^{+}(y ; t)$, and an integral form of the moments of $Y^{+}(t)$.

Corollary 2.1. (i) The density of $Y^{+}(t)$ is given by

$$
\begin{aligned}
\psi^{+}(y ; t)= & f_{U}(y)-\sum_{n=1}^{+\infty}\left[f_{U}^{(n)}(y)-f_{U}^{(n+1)}(y)\right] F_{T}^{(n)}(t-y) \\
& +\sum_{n=1}^{\infty}\left[F_{U}^{(n)}(y)-F_{U}^{(n+1)}(y)\right] f_{T}^{(n)}(t-y), \quad 0<y<t .
\end{aligned}
$$

(ii) The moments of $Y^{+}(t), t>0$, are

$$
\mathrm{E}\left[Y^{+}(t)^{n}\right]=n \int_{0}^{t} x^{n-1} F_{T_{U}}(t-x ; x) \mathrm{d} x, \quad n=1,2, \ldots
$$

The distribution of $Y^{-}(t)$ and its moments can be derived in a similar manner. 


\section{The distribution of $X(t)$}

Aiming to determine the distribution of $X(t)$, we recall that $X(t)=Y^{+}(t)-Y^{-}(t)$, where $Y^{+}(t)$ and $Y^{-}(t)$ are defined in (1.1) and (1.2). Hence, we have

$$
\mathrm{P}[X(t) \leq x]=\mathrm{E}\left[\mathrm{P}\left[Y^{-}(t) \geq Y^{+}(t)-x \mid Y^{+}(t)\right]\right] .
$$

We have therefore to derive the conditional probability $\mathrm{P}\left[Y^{-}(t) \geq z \mid Y^{+}(t)=y\right], 0<y<t$. Note that $\mathrm{P}\left[Y^{-}(t)=0 \mid Y^{+}(t)=t\right]=1$. For deriving this conditional probability, define the compound process

$$
S_{V}(\omega)=\sum_{n=0}^{N_{V}(\omega)} W_{n}, \quad \omega>0,
$$

where $W_{0}=0$. Moreover, we let $S_{n}^{V}=\sum_{i=1}^{n} V_{i}$ and $N_{V}(\omega)=\max \left\{n \geq 0: S_{n}^{V} \leq \omega\right\}$. The $\mathrm{CDF}$ of $S_{V}(\omega)$ is

$$
F_{S_{V}}(y ; \omega)=\mathrm{P}\left[S_{V}(\omega) \leq y\right]=\sum_{n=0}^{\infty}\left[F_{V}^{(n)}(\omega)-F_{V}^{(n+1)}(\omega)\right] F_{W}^{(n)}(y) .
$$

Note that $F_{S_{V}}(0 ; \omega)=1-F_{V}(\omega)$. We set, for $t>y$,

$$
\tilde{Y}(t-y)=\inf \left\{\omega>y: S_{V}(\omega-y) \geq t-\omega\right\}
$$

(see Figure 3 for an example). Since $\left\{U_{i}\right\},\left\{V_{i}\right\}$, and $\left\{W_{i}\right\}$ are mutually independent, and $S_{V}(\omega)$ is an increasing process, we have $\tilde{Y}(t-y) \stackrel{\text { D }}{=} Y^{-}(t)+y$, where $\stackrel{\text { D }}{=}$ ' denotes equality in distribution. Moreover, for $0<z<t-y$, we have

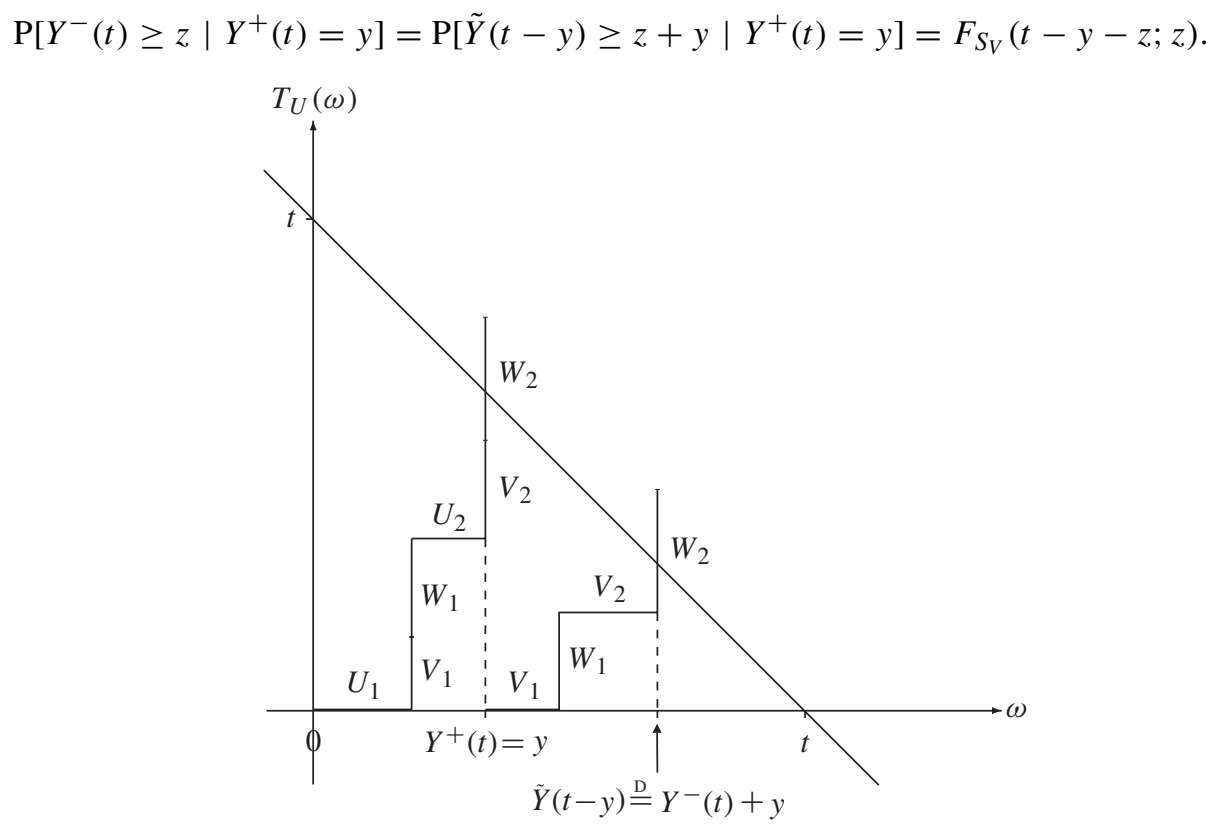

FIGURE 3: The stopping times $Y^{+}(t)$ and $\tilde{Y}(t-y)$. 
In the following theorems we obtain the general form of the distribution function of $X(t)$, denoted as $F_{X}(x ; t)$, and its mean.

Theorem 3.1. The CDF of $X(t)$ is given by

$$
F_{X}(x ; t)=\mathrm{P}\left[Y^{+}(t) \leq x\right]+\int_{x}^{(t+x) / 2} \psi^{+}(y ; t) F_{S_{V}}(t-2 y+x ; y-x) \mathrm{d} y
$$

if $0<x \leq t$ and by

$$
F_{X}(x ; t)=\int_{0}^{(t+x) / 2} \psi^{+}(y ; t) F_{S_{V}}(t-2 y+x ; y-x) \mathrm{d} y
$$

if $-t<x \leq 0$.

Proof. According to (3.1) we have

$$
F_{X}(x ; t)=\int_{0}^{\beta} \mathrm{P}\left[Y^{-}(t) \geq y-x \mid Y^{+}(t)=y\right] \psi^{+}(y ; t) \mathrm{d} y,
$$

but $\mathrm{P}\left[Y^{-}(t)>t-y \mid Y^{+}(t)=y\right]=0$. Hence, for the conditional probability of $\left\{Y^{-}(t) \geq\right.$ $y-x\}$, given that $\left\{Y^{+}(t)=y\right\}$ is positive, we must have $\beta=(t+x) / 2$. Now, $\mathrm{P}\left[Y^{-}(t)>\right.$ $\left.0 \mid Y^{+}(t)=y\right]=1$. Accordingly,

$$
F_{X}(x ; t)=\int_{0}^{x} \psi^{+}(y ; t) \mathrm{d} y+\int_{x}^{(t+x) / 2} \psi^{+}(y ; t) F_{S_{V}}(t-2 y+x ; y-x) \mathrm{d} y .
$$

Also, $\int_{0}^{x} \psi^{+}(y ; t) \mathrm{d} y=\mathrm{P}\left[Y^{+}(t) \leq x\right]$. This proves (3.3). Finally, for all $-t<x \leq 0$, $y-x>0$ for all $y$. This yields (3.4).

Theorem 3.2. For all $t>0$, we have

$$
\begin{aligned}
\mathrm{E}[X(t)]= & \int_{0}^{t} F_{T_{U}}(t-x ; x) \mathrm{d} x-\int_{0}^{t / 2} \psi^{+}(y ; t) \int_{0}^{y} F_{S_{V}}(t-2 y+x ; y-x) \mathrm{d} x \mathrm{~d} y \\
& -\int_{t / 2}^{t} \psi^{+}(y ; t) \int_{2 y-t}^{y} F_{S_{V}}(t-2 y+x ; y-x) \mathrm{d} x \mathrm{~d} y \\
& -\int_{0}^{t / 2} \psi^{+}(y ; t) \int_{2 y-t}^{0} F_{S_{V}}(t-2 y+x ; y-x) \mathrm{d} x \mathrm{~d} y .
\end{aligned}
$$

Proof. It is well known that

$$
\mathrm{E}[X(t)]=\int_{0}^{t} \bar{F}_{X}(x ; t) \mathrm{d} x-\int_{-t}^{0} F_{X}(x ; t) \mathrm{d} x,
$$

where $\bar{F}_{X}(x ; t)=1-F_{X}(x ; t)$. According to (3.3), for $x>0$, we have

$$
\bar{F}_{X}(x ; t)=\mathrm{P}\left[Y^{+}(t)>x\right]-\int_{x}^{(t+x) / 2} \psi^{+}(y ; t) F_{S_{V}}(t-2 y+x ; y-x) \mathrm{d} y .
$$

Moreover,

$$
\int_{0}^{t} \mathrm{P}\left[Y^{+}(t)>x\right] \mathrm{d} x=\int_{0}^{t} F_{T_{U}}(t-x ; x) \mathrm{d} x .
$$


Also,

$$
\begin{aligned}
\int_{0}^{t} \int_{x}^{(t+x) / 2} \psi^{+}(y ; t) F_{S_{V}}(t-2 y+x ; y-x) \mathrm{d} y \mathrm{~d} x \\
=\int_{0}^{t / 2} \psi^{+}(y ; t) \int_{0}^{y} F_{S_{V}}(t-2 y+x ; y-x) \mathrm{d} x \mathrm{~d} y \\
\quad+\int_{t / 2}^{t} \psi^{+}(y ; t) \int_{2 y-t}^{y} F_{S_{V}}(t-2 y+x ; y-x) \mathrm{d} x \mathrm{~d} y .
\end{aligned}
$$

Finally,

$$
\begin{aligned}
\int_{-t}^{0} \int_{0}^{(t+x) / 2} \psi^{+}(y ; t) F_{S_{V}}(t-2 y+x ; y-x) \mathrm{d} y \mathrm{~d} x \\
\quad=\int_{0}^{t / 2} \psi^{+}(y ; t) \int_{2 y-t}^{0} F_{S_{V}}(t-2 y+x ; y-x) \mathrm{d} x \mathrm{~d} y .
\end{aligned}
$$

Substituting (3.7)-(3.9) into (3.6) we have (3.5).

\section{Special cases}

In this section we consider some cases of interest arising when the random times $U_{i}, V_{i}$, and $W_{i}$ have exponential distribution with equal rates, unequal rates, and linear increasing rates. We obtain explicit expressions for the density $\psi^{+}(y ; t)$ and, if possible, for the distribution function and the mean of $X(t)$.

\subsection{Exponentially distributed times with equal rates}

We assume that $U_{i}, V_{i}$, and $W_{i}$ are i.i.d. exponentially distributed random variables with parameter $\lambda$. Hence, recalling (2.6) we have, for $n=0,1, \ldots$,

$$
p_{n}(t)=\mathrm{e}^{-\lambda t} \frac{(\lambda t)^{n}}{n !}, \quad t \geq 0
$$

Proposition 4.1. If $U_{i}, V_{i}$, and $W_{i}$ are exponentially distributed with parameter $\lambda$, then the density of $Y^{+}(t)$, for $0<y<t$, is given by

$$
\begin{aligned}
\psi^{+}(y ; t)=\lambda \mathrm{e}^{-\lambda t} & \left\{\lambda^{2} y(t-y)_{0} F_{2}\left[\{\} ; \frac{3}{2}, 2 ; \frac{1}{4} \lambda^{3} y(t-y)^{2}\right]\right. \\
& +\lambda(t-y)_{0} F_{2}\left[\{\} ; 1, \frac{3}{2} ; \frac{1}{4} \lambda^{3} y(t-y)^{2}\right] \\
& \left.+{ }_{0} F_{2}\left[\{\} ; 1, \frac{1}{2} ; \frac{1}{4} \lambda^{3} y(t-y)^{2}\right]\right\},
\end{aligned}
$$

where ${ }_{0} F_{2}[\{\} ; c, d ; z]$ is the generalized hypergeometric function.

Proof. Equations (2.5) and (4.1) yield

$$
F_{T_{U}}(y ; t)=\mathrm{e}^{-\lambda t} \sum_{n=0}^{+\infty} \frac{(\lambda t)^{n}}{n !} \frac{\gamma(2 n, \lambda y)}{(2 n-1) !}=1-\mathrm{e}^{-\lambda(t+y)} \sum_{n=1}^{+\infty} \frac{(\lambda t)^{n}}{n !} \sum_{j=0}^{2 n-1} \frac{(\lambda y)^{j}}{j !},
$$

where $\gamma(s, x)$ denotes the lower incomplete gamma function, and use of the following relation has been made:

$$
F_{T}^{(n)}(y)=\frac{\gamma(2 n, \lambda y)}{(2 n-1) !}=1-\mathrm{e}^{-\lambda y} \sum_{j=0}^{2 n-1} \frac{(\lambda y)^{j}}{j !} .
$$


The corresponding probability density function of (4.3) is given by

$$
f_{T_{U}}(y ; t)=\lambda \mathrm{e}^{-\lambda(t+y)} \sum_{n=1}^{+\infty} \frac{(\lambda t)^{n}}{n !} \frac{(\lambda y)^{2 n-1}}{(2 n-1) !}=\mathrm{e}^{-\lambda(t+y)} \lambda^{3} t y_{0} F_{2}\left[\{\} ; \frac{3}{2}, 2 ; \frac{1}{4} \lambda^{3} t y^{2}\right]
$$

for $y>0$. From this we have

$$
\mathrm{P}\left[Y^{+}(t)>y\right]=1-\mathrm{e}^{-\lambda t} \sum_{n=1}^{+\infty} \frac{(\lambda y)^{n}}{n !} \sum_{j=0}^{2 n-1} \frac{[\lambda(t-y)]^{j}}{j !} .
$$

Hence, after some calculations we obtain the density (4.2).

In Figure 4 we present the density $\psi^{+}(y ; t)$, expressed in Proposition 4.1, for various choices of $\lambda$ and $t$.

Proposition 4.2. If $U_{i}, V_{i}$, and $W_{i}$ are exponentially distributed with parameter $\lambda$, then the distribution function of $X(t)$ is given by

$$
\begin{aligned}
F_{X}(x ; t)= & \mathrm{e}^{-\lambda x} \sum_{n=1}^{+\infty} \frac{(\lambda x)^{n}}{n !} \frac{\Gamma(2 n, \lambda(t-x))}{(2 n-1) !} \\
+ & \mathrm{e}^{-\lambda t} \sum_{n=1}^{+\infty} \sum_{k=0}^{n} \frac{(\lambda x)^{n-1-k}}{(n-k) !} \frac{[\lambda(t-x)]^{k+1}}{2^{k+1} k !} \\
& \times \sum_{l=0}^{2 n-1} \frac{(-1)^{l}}{2^{l} l !(2 n-1-l) !} \sum_{s=0}^{+\infty}[\lambda(t-x)]^{s} \sum_{r=0}^{s} \frac{[\lambda(t-x)]^{r}}{2^{r} r !} \frac{(k+l+r) !}{(k+l+r+s+1) !} \\
& \times{ }_{1} F_{1}\left[k+l+r+1 ; k+l+r+s+2 ; \frac{1}{2} \lambda(t-x)\right] \\
& \quad \times\left\{\lambda x \mathrm{e}^{-\lambda(t-x)}[\lambda(t-x)]^{2 n-1}\right. \\
& \left.\quad+(n-k-\lambda x)[\lambda(t-x)]^{l} \Gamma(2 n-l, \lambda(t-x))\right\}
\end{aligned}
$$

for $0<x \leq t$ and by

$$
\begin{aligned}
F_{X}(x ; t)= & \mathrm{e}^{-\lambda t} \sum_{n=1}^{+\infty} \frac{[\lambda(t+x)]^{n+1}}{2^{n+1} n !} \sum_{s=0}^{+\infty}[\lambda(t+x)]^{s} \sum_{r=0}^{s} \sum_{k=0}^{r} \frac{(-\lambda x)^{r-k}}{(r-k) !} \frac{[\lambda(t+x)]^{k}}{2^{k} k !} \\
& \times \sum_{l=0}^{2 n-1} \frac{(-1)^{l}[\lambda(t+x)]^{l}}{2^{l} l !(2 n-1-l) !} \frac{(k+l+n) !}{(k+l+n+s+1) !} \\
& \times{ }_{1} F_{1}\left[k+l+n+1 ; k+l+n+s+2 ; \frac{1}{2} \lambda(t+x)\right] \\
& \times\left\{\mathrm{e}^{-\lambda t}(\lambda t)^{2 n-l-1}-\Gamma(2 n-l, \lambda t)\right\} \\
+ & \mathrm{e}^{-\lambda t} \sum_{n=1}^{+\infty} \frac{[\lambda(t+x)]^{n}}{2^{n}(n-1) !} \sum_{s=0}^{+\infty}[\lambda(t+x)]^{s} \sum_{r=0}^{s} \sum_{k=0}^{r} \frac{(-\lambda x)^{r-k}}{(r-k) !} \frac{[\lambda(t+x)]^{k}}{2^{k} k !} \\
& \times \sum_{l=0}^{2 n-1} \frac{(-1)^{l}[\lambda(t+x)]^{l}}{2^{l} l !} \frac{\Gamma(2 n-l, \lambda t)}{(2 n-l-1) !} \frac{(k+l+n-1) !}{(k+l+n+s) !} \\
& \times{ }_{1} F_{1}\left[k+l+n ; k+l+n+s+1 ; \frac{1}{2} \lambda(t+x)\right]
\end{aligned}
$$

for $-t<x \leq 0$, where $\Gamma(s, x)$ is the upper incomplete gamma function and ${ }_{1} F_{1}[a ; b ; z]$ is the confluent hypergeometric function of the first kind. 

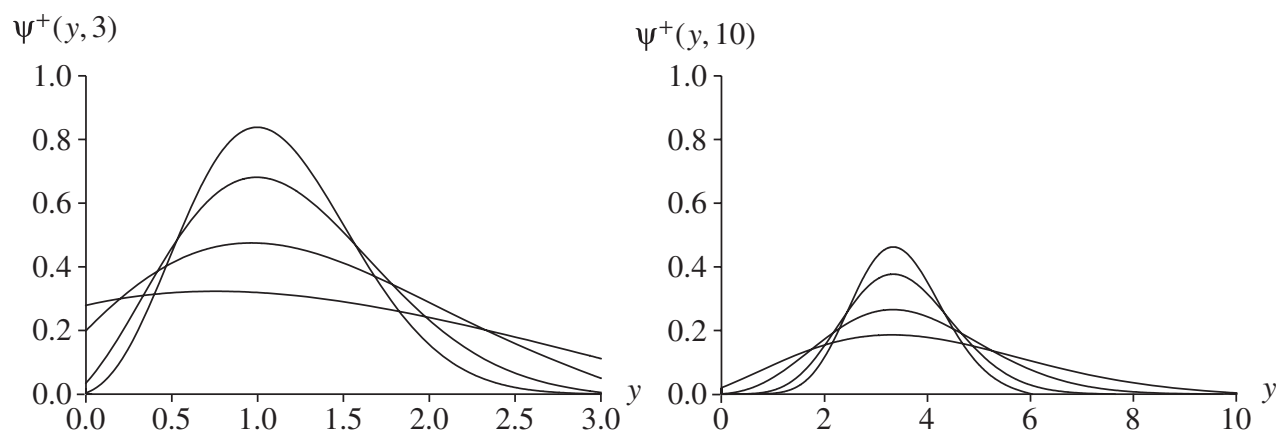

Figure 4: Density (4.2) for $\lambda=0.5,1,2,3$ (from bottom to top near the mode) for $t=3$ (left) and $t=10($ right $)$.

Proof. In the present special case

$$
F_{S_{V}}(y ; \omega)=\sum_{n=0}^{+\infty} p_{n}(\omega)[1-P(n-1, \lambda y)]=\sum_{n=0}^{+\infty} p_{n}(y) P(n, \lambda \omega),
$$

where $p_{n}(\cdot)$ is given in (4.1) and we have set

$$
P(n, x)=\mathrm{e}^{-x} \sum_{k=0}^{n} \frac{x^{k}}{k !}
$$

Thus, according to (3.2),

$$
\mathrm{P}\left[Y^{-}(t) \geq z \mid Y^{+}(t)=y\right]=\sum_{n=0}^{+\infty} p_{n}(t-y-z) P(n, \lambda z) .
$$

Hence, due to Theorem 3.1, (4.2), and (4.4), making use of Equations 3.383.1 and 3.384.2 of [10], and recalling that Whittaker's function is given (cf. [11] or Equation 13.1.32 of [1]) by $M_{l, m}(z)=z^{m+1 / 2} \mathrm{e}^{-z / 2}{ }_{1} F_{1}\left[m-l+\frac{1}{2} ; 2 m+1 ; z\right]$, the proof follows after some straightforward calculations.

Some plots of the distribution functions obtained in Proposition 4.2 are given in Figure 5.
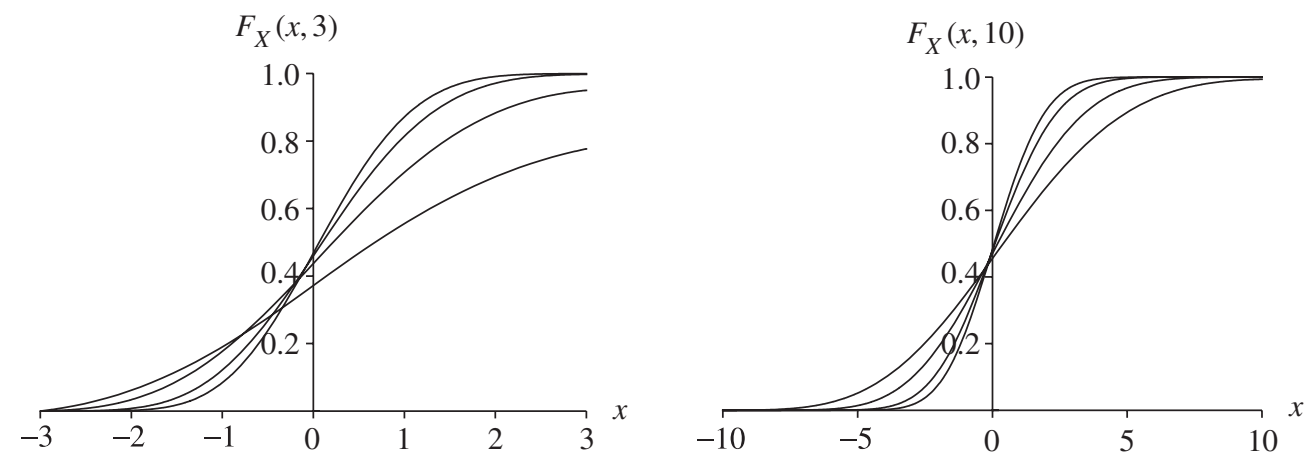

FIGURE 5: Distribution functions (4.5) and (4.6) for $\lambda=\frac{1}{2}, 1,2,3$ (from bottom to top when $x>0$ ) for $t=3$ (left) and $t=10$ (right). 
Proposition 4.3. If $U_{i}, V_{i}$, and $W_{i}$ are exponentially distributed with parameter $\lambda$, then the mean of $X(t)$ is given by

$$
\begin{aligned}
& \mathrm{E}[X(t)]=t-t \mathrm{e}^{-\lambda t} \sum_{n=1}^{+\infty} \sum_{j=0}^{2 n-1} \frac{(\lambda t)^{n+j}}{(j+n+1) !} \\
& +t \mathrm{e}^{-2 \lambda t} \sum_{n=1}^{+\infty} \frac{(\lambda t)^{n}}{2^{n}} \sum_{s=0}^{+\infty}(\lambda t)^{s} \sum_{r=0}^{s} \frac{(\lambda t)^{r}}{2^{r} r !} \sum_{h=0}^{+\infty} \frac{(\lambda t)^{h}}{2^{h} h !} \\
& \times\left\{-\frac{(\lambda t)^{2 n}}{2^{n}(2 n-1) !} \sum_{k=0}^{n} \frac{1}{2^{k} k !} \frac{(h+k+r) !}{(h+k+r+s+1) !} \frac{(h+k+2 n+r+s) !}{(h+3 n+r+s+1) !}\right. \\
& \times{ }_{2} F_{1}[1-2 n, 1+s ; h+k+r+s+2 ;-1] \\
& \times{ }_{1} F_{1}[1-k+n ; 2+h+3 n+r+s ; \lambda t] \\
& +\frac{1}{n !} \sum_{k=0}^{2 n-1} \frac{(\lambda t)^{k+1}}{2^{k+1} k !} \frac{1}{(h+k+n+r+s+2)} \frac{(h+n+r) !}{(h+n+r+s+1) !} \\
& \times{ }_{2} F_{1}[-k, 1+s ; h+n+r+s+2 ;-1] \\
& \times{ }_{1} F_{1}[1 ; 3+h+k+n+r+s ; \lambda t] \\
& +\sum_{k=0}^{n-1} \frac{2^{n-1-k}}{k !} \sum_{j=0}^{2 n-1} \frac{(\lambda t)^{j}}{2^{j} j !} \frac{(h+k+r) !}{(h+k+r+s+1) !} \frac{(h+k+j+r+s+1) !}{(h+j+n+r+s+2) !} \\
& \times{ }_{2} F_{1}[-j, 1+s ; h+k+r+s+2 ;-1] \\
& \times\left(\lambda t_{1} F_{1}[1-k+n ; 3+h+j+n+r+s ; \lambda t]\right. \\
& -(2+h+j+n+r+s) \\
& \left.\left.\times{ }_{1} F_{1}[-k+n ; 2+h+j+n+r+s ; \lambda t]\right)\right\} \\
& -t \mathrm{e}^{-\lambda t} \sum_{n=1}^{+\infty} \frac{(\lambda t)^{n}}{2^{n+1} n !} \sum_{s=0}^{+\infty}(\lambda t)^{s} \sum_{r=0}^{s}(\lambda t)^{r} \sum_{k=0}^{r} \frac{1}{2^{k} k !} \\
& \times \sum_{l=0}^{2 n-1} \frac{(-1)^{l}}{2^{l} l !} \frac{(k+l+n-1) !}{(2 n-1-l) !(2+l+n+r+s) !} \\
& \times\left\{\mathrm{e}^{-\lambda t}(\lambda t)^{2 n}(k+l+n){ }_{1} F_{1}\left[k+l+n+1 ; l+n+r+s+3 ; \frac{1}{2} \lambda t\right]\right. \\
& -(\lambda t)^{l} \Gamma(2 n-1, \lambda t) \\
& \times\left(\lambda t(k+l+n){ }_{1} F_{1}\left[k+l+n+1 ; l+n+r+s+3 ; \frac{1}{2} \lambda t\right]\right. \\
& -2 n(2+l+n+r+s) \\
& \left.\left.\times{ }_{1} F_{1}\left[k+l+n ; l+n+r+s+2 ; \frac{1}{2} \lambda t\right]\right)\right\} \text {. }
\end{aligned}
$$

In Figure 6 we present some plots of $\mathrm{E}[X(t)]$.

\subsection{Exponentially distributed times with unequal rates}

We suppose that $U_{i}, V_{i}$, and $W_{i}$ have exponential distributions. Precisely, let $U_{i}, V_{i}$, and $W_{i}$ be i.i.d. random variables with respective parameters $\lambda, \mu$, and $\nu$. The three parameters are 


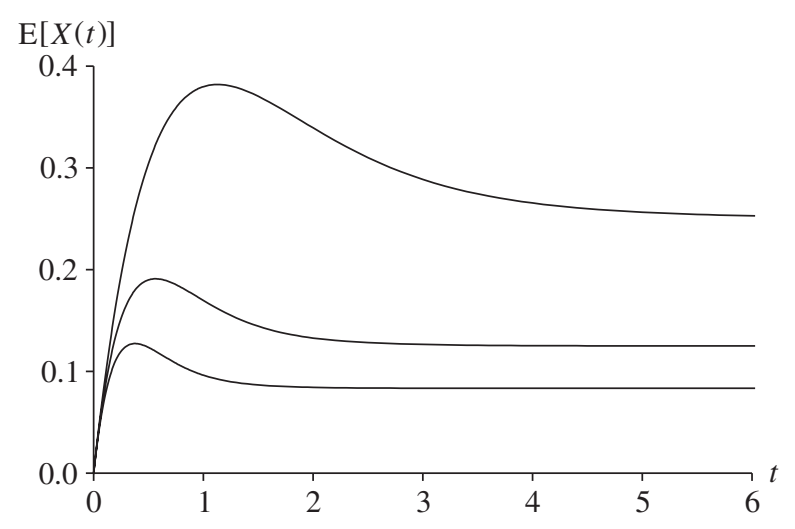

Figure 6: Mean (4.9) for $\lambda=1,2,3$ (from top to bottom).

all unequal. Hence, (4.1) holds. Moreover, due to (2.2),

$$
F_{T}(t)=\frac{\mu\left(1-\mathrm{e}^{-v t}\right)}{\mu-v}+\frac{v\left(1-\mathrm{e}^{-\mu t}\right)}{v-\mu}, \quad t \geq 0 .
$$

The density $f_{T}^{(n)}(t)$ can be obtained as the convolution of two gamma densities with parameters $\mu$ and $v$, so that

$$
\begin{aligned}
f_{T}^{(n)}(t) & =\int_{0}^{t} \frac{\mu \mathrm{e}^{-\mu x}(\mu x)^{n-1}}{(n-1) !} \frac{v \mathrm{e}^{-v(t-x)}[v(t-x)]^{n-1}}{(n-1) !} \mathrm{d} x \\
& =\frac{(\mu \nu)^{n} t^{2 n-1}}{(2 n-1) !} \mathrm{e}^{-v t}{ }_{1} F_{1}[n ; 2 n ;(\nu-\mu) t], \quad t>0 .
\end{aligned}
$$

From (4.10) we also have

$$
\begin{aligned}
F_{T}^{(n)}(t)= & \left(1-\frac{\mu}{\mu-v}\right)^{n} \sum_{k=0}^{n-1}\left(\begin{array}{c}
n+k-1 \\
k
\end{array}\right)\left(\frac{\mu}{\mu-v}\right)^{k}[1-P(n-k, \mu t)] \\
& +\left(1-\frac{v}{v-\mu}\right)^{n} \sum_{k=0}^{n-1}\left(\begin{array}{c}
n+k-1 \\
k
\end{array}\right)\left(\frac{v}{v-\mu}\right)^{k}[1-P(n-k, v t)],
\end{aligned}
$$

where $P(n, x)$ is defined in (4.8).

Proposition 4.4. If $U_{i}, V_{i}$, and $W_{i}$ are exponentially distributed with parameters $\lambda, \mu$, and $\nu$, respectively, then the density of $Y^{+}(t)$ is given, for $0<y<t$, by

$$
\begin{aligned}
\psi^{+}(y ; t)= & \lambda \mathrm{e}^{-\lambda y} \\
- & \lambda \mathrm{e}^{-\lambda y} \sum_{n=1}^{\infty}\left[\frac{(\lambda y)^{n-1}}{(n-1) !}-\frac{(\lambda y)^{n}}{n !}\right] \\
& \times\left\{\left(1-\frac{\mu}{\mu-v}\right)^{n} \sum_{k=0}^{n-1}\left(\begin{array}{c}
n+k-1 \\
k
\end{array}\right)\left(\frac{\mu}{\mu-v}\right)^{k}[1-P(n-k, \mu(t-y))]\right. \\
& \left.+\left(1-\frac{v}{v-\mu}\right)^{n} \sum_{k=0}^{n-1}\left(\begin{array}{c}
n+k-1 \\
k
\end{array}\right)\left(\frac{v}{v-\mu}\right)^{k}[1-P(n-k, v(t-y))]\right\}
\end{aligned}
$$




$$
\begin{aligned}
+\mathrm{e}^{-\lambda y} \sum_{n=1}^{\infty}\left[\frac{(\lambda y)^{n}}{n !}\right] & \left\{\frac{\mathrm{e}^{-\mu(t-y)}(-2 \mu \nu)^{n}}{2(n-1) !(\mu-v)^{2 n-1}} \theta_{n-1}\left[\frac{(\mu-v)(t-y)}{2}\right]\right. \\
& \left.+\frac{\mathrm{e}^{-v(t-y)}(-2 \mu \nu)^{n}}{2(n-1) !(\nu-\mu)^{2 n-1}} \theta_{n-1}\left[\frac{(\nu-\mu)(t-y)}{2}\right]\right\},
\end{aligned}
$$

where

$$
\theta_{n}(x)=\sum_{k=0}^{n} \frac{(2 n-k) !}{(n-k) ! k !} \frac{x^{k}}{2^{n-k}}
$$

is the reverse Bessel polynomial (see [2] for instance).

Proof. Recalling (2.5), we obtain

$$
\begin{gathered}
F_{T_{U}}(y ; t)=\mathrm{e}^{-\lambda t}+\mathrm{e}^{-\lambda t} \sum_{n=1}^{+\infty} \frac{(\lambda t)^{n}}{n !}\left(1-\frac{\mu}{\mu-v}\right)^{n} \sum_{k=0}^{n-1}\left(\begin{array}{c}
n+k-1 \\
k
\end{array}\right)\left(\frac{\mu}{\mu-v}\right)^{k} \\
\times[1-P(n-k, \mu y)] \\
+\mathrm{e}^{-\lambda t} \sum_{n=1}^{+\infty} \frac{(\lambda t)^{n}}{n !}\left(1-\frac{v}{v-\mu}\right) \sum_{k=0}^{n-1}\left(\begin{array}{c}
n+k-1 \\
k
\end{array}\right)\left(\frac{v}{v-\mu}\right)^{k} \\
\times[1-P(n-k, v y)]
\end{gathered}
$$

with $F_{T_{U}}(0 ; t)=\mathrm{e}^{-\lambda t}$ and, for $y>0$, the density of $F_{T_{U}}(\cdot)$ is

$$
\begin{aligned}
f_{T_{U}}(y ; t)= & \frac{\mathrm{e}^{-\mu y}}{2} \sum_{n=1}^{+\infty} \frac{\mathrm{e}^{-\lambda t}(\lambda t)^{n}}{n !} \frac{(-2 \mu \nu)^{n}}{(n-1) !(\mu-\nu)^{2 n-1}} \theta_{n-1}\left[\frac{(\mu-v) y}{2}\right] \\
& +\frac{\mathrm{e}^{-v y}}{2} \sum_{n=1}^{+\infty} \frac{\mathrm{e}^{-\lambda t}(\lambda t)^{n}}{n !} \frac{(-2 \mu \nu)^{n}}{(n-1) !(v-\mu)^{2 n-1}} \theta_{n-1}\left[\frac{(\nu-\mu) y}{2}\right] .
\end{aligned}
$$

Finally, due to (2.7), the density of $Y^{+}(t)$, on $(0, t)$, is given by (4.11).

We remark that the following symmetry property of $\psi^{+}(y ; t)$ immediately follows from (4.11):

$$
\left.\psi^{+}(y ; t)\right|_{\mu=c_{1}, \nu=c_{2}}=\left.\psi^{+}(y ; t)\right|_{\mu=c_{2}, \nu=c_{1}}
$$

for all $0<y<t$ and $c_{1}, c_{2}>0$.

Some plots of the density $\psi^{+}(y ; t)$, obtained in Proposition 4.4, are given in Figure 7 for various choices of $\lambda, \mu, v$, and $t$.

Applying reasoning similar to that used in the proof of Proposition 4.2, and recalling that in the present case

$$
F_{S_{V}}(y ; \omega)=\mathrm{e}^{-v y} \sum_{n=0}^{+\infty} \frac{(v y)^{n}}{n !} P(n, \mu \omega),
$$

it is possible to obtain the distribution function of $X(t)$ by means of straightforward calculations. However, we omit the expression since it is very cumbersome. Some plots are shown in Figures 8 and 9. 


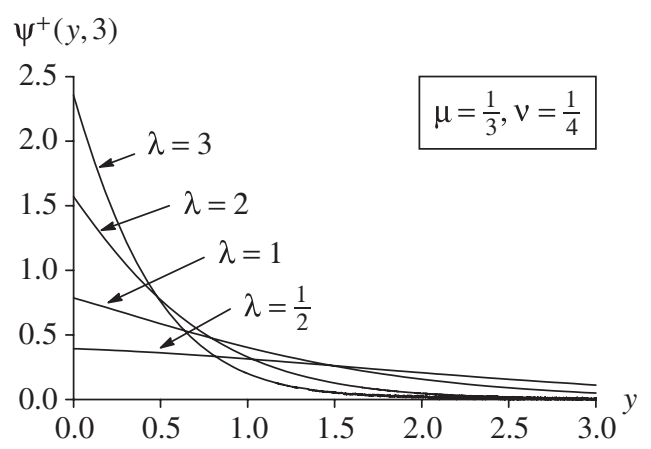

$$
\psi^{+}(y, 10)
$$
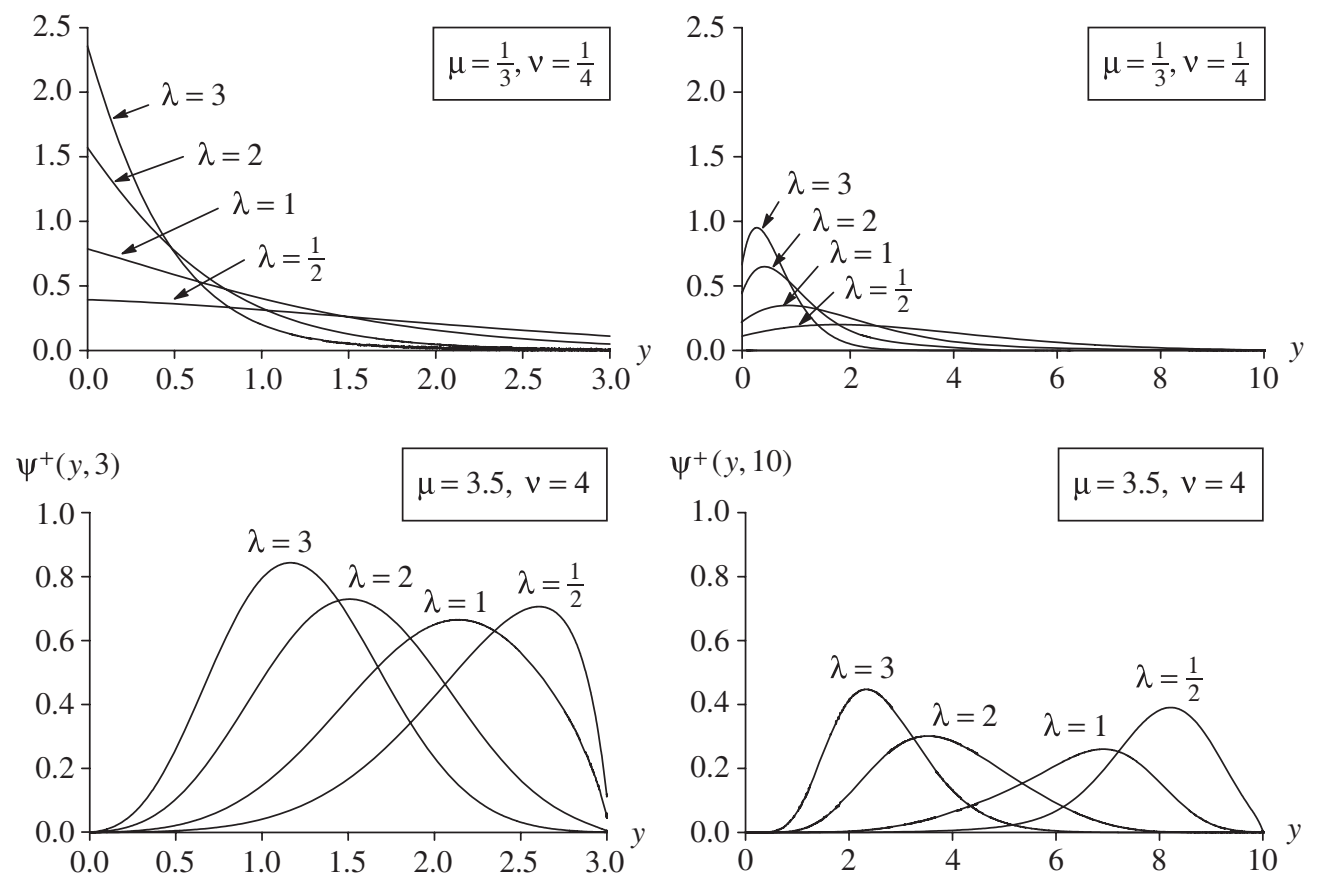

Figure 7: Density (4.11) for $t=3$ (left) and $t=10$ (right); the values of $\lambda, \mu$, and $v$ are indicated.
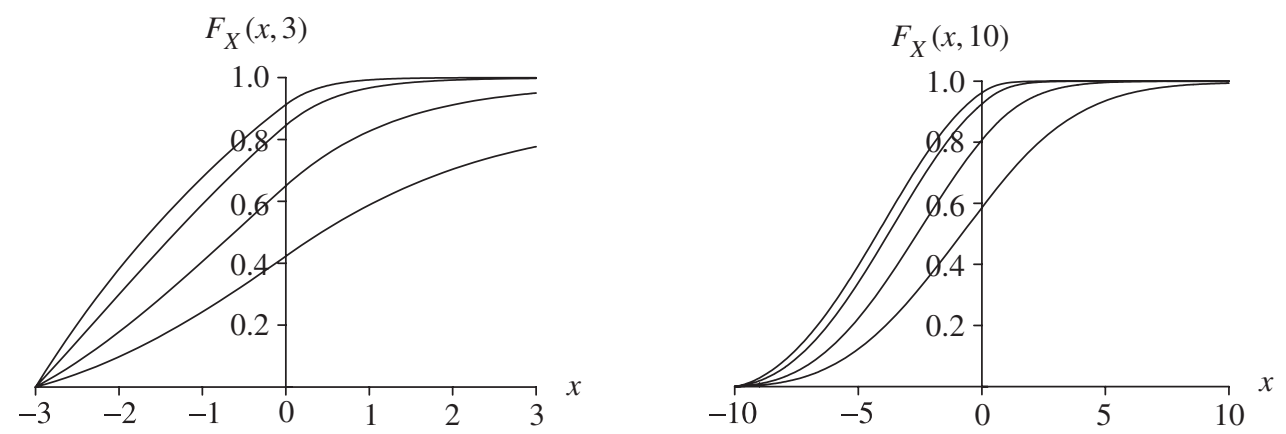

FIgURE 8: The distribution function of $X(t)$ given in Proposition 4.4, with $\lambda=\frac{1}{2}, 1,2,3$ (from bottom to top), $\mu=\frac{1}{3}$, and $v=\frac{1}{4}$, for $t=3$ (left) and $t=10$ (right).

\subsection{Damped process}

In this section we assume that $U_{i}, V_{i}$, and $W_{i}$ are independent random variables, exponentially distributed with parameter $\lambda i(i=1,2, \ldots)$. A similar choice has been considered by Di Crescenzo and Martinucci [6] and Di Crescenzo et al. [8], who studied a damped telegraph process and a damped geometric telegraph process, respectively. Since the parameters $\lambda i$ are linear increasing in $i$, the process $X(t)$ exhibits a damped behavior, in the sense that its sample paths are composed of line segments that become stochastically smaller and smaller. 

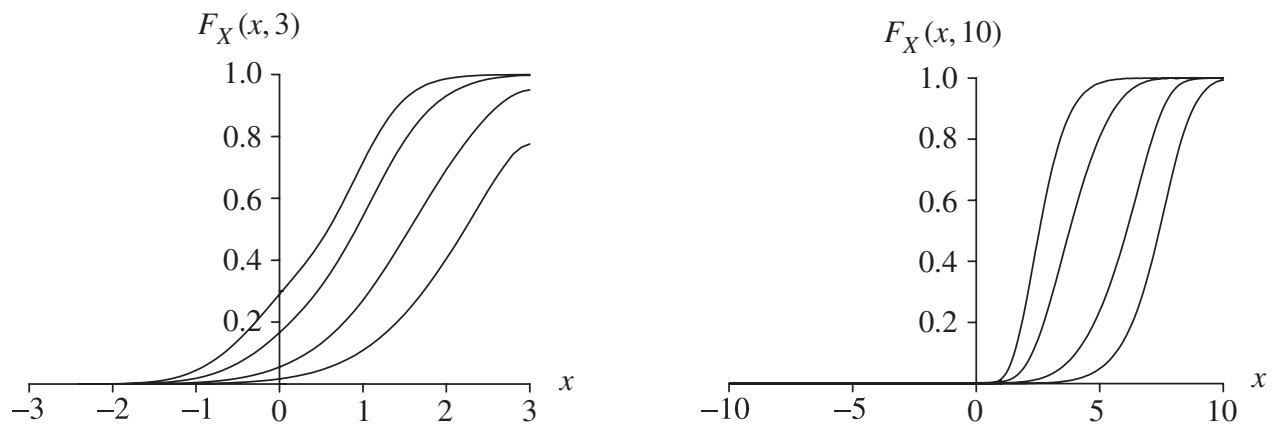

FIGURE 9: Same as Figure 8, with $\mu=3.5$ and $v=4$.

The assumption that the parameters of $U_{i}, V_{i}$, and $W_{i}$ are $\lambda i$ implies that the random times separating consecutive velocity reversals have the same distribution of the intertimes of a simple birth process (see [17] for instance).

Due to Equation (11) of [6], the density and the distribution function of the $n$-fold convolution $U^{(n)}$ for $n \geq 1$ are given by

$$
\begin{aligned}
& f_{U}^{(n)}(x)=n \lambda \mathrm{e}^{-\lambda x}\left(1-\mathrm{e}^{-\lambda x}\right)^{n-1}, \\
& F_{U}^{(n)}(x)=\left(1-\mathrm{e}^{-\lambda x}\right)^{n} .
\end{aligned}
$$

We now set

$$
\zeta_{k}(j, n ; z)={ }_{2} F_{1}\left[1, j+k ; n+1 ; 1-\mathrm{e}^{\lambda z}\right],
$$

where ${ }_{2} F_{1}[a, b ; c ; z]$ denotes the Gauss hypergeometric function.

Proposition 4.5. If $U_{i}, V_{i}$, and $W_{i}$ are exponentially distributed with parameters $\lambda i, i=$ $1,2, \ldots$, then the density of $Y^{+}(t)$ is given, for $0<y<t$, by

$$
\begin{aligned}
& \psi^{+}(y ; t)=\lambda \mathrm{e}^{-\lambda y}- \lambda \mathrm{e}^{-\lambda y}\left(1-\mathrm{e}^{-\lambda(t-y)}\right) \\
& \times \sum_{n=1}^{\infty}\left[\left(1-\mathrm{e}^{-\lambda y}\right)\left(1-\mathrm{e}^{-\lambda(t-y)}\right)\right]^{n-1}\left[(n+1) \mathrm{e}^{-\lambda y}-1\right] \\
& \times \sum_{j=0}^{n}\left(\begin{array}{c}
n \\
j
\end{array}\right)(-1)^{j} \zeta_{0}(j, n ; t-y) \\
&+\lambda \sum_{n=1}^{\infty} n\left[\mathrm{e}^{-\lambda y}\left(1-\mathrm{e}^{-\lambda y}\right)\left(1-\mathrm{e}^{-\lambda(t-y)}\right)\right]^{n} \sum_{j=0}^{n-1}\left(\begin{array}{c}
n-1 \\
j
\end{array}\right)(-1)^{j} \zeta_{1}(j, n ; t-y) .
\end{aligned}
$$

Proof. Due to Equation 3.196.1 of [10], the following identity holds:

$$
\begin{aligned}
& \int_{0}^{z} f_{V}^{(n)}(v) f_{W}^{(n)}(t-y-v) \mathrm{d} v \\
& \quad=n \lambda\left(1-\mathrm{e}^{-\lambda z}\right)^{n} \sum_{j=0}^{n-1}\left(\begin{array}{c}
n-1 \\
j
\end{array}\right)(-1)^{j} \mathrm{e}^{-\lambda(j+1)(t-y-z)} \zeta_{1}(j, n ; z) .
\end{aligned}
$$



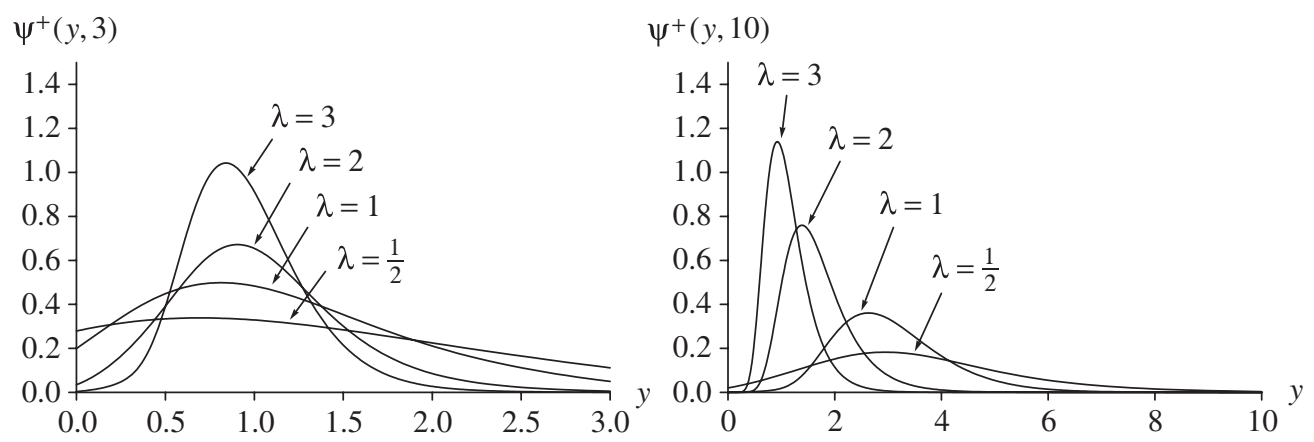

Figure 10: Density (4.13) for $t=3$ (left) and $t=10$ (right); the values of $\lambda$ are indicated.

Making use of (4.14) and recalling (2.1), we thus obtain

$$
\begin{aligned}
f_{T}^{(n)}(t) & =n^{2} \lambda^{2} \mathrm{e}^{-\lambda t} \int_{0}^{t}\left[\left(1-\mathrm{e}^{-\lambda x}\right)\left(1-\mathrm{e}^{-\lambda(t-x)}\right)\right]^{n-1} \mathrm{~d} x \\
& =n^{2} \lambda^{2} \mathrm{e}^{-\lambda t} \sum_{j=0}^{n-1}\left(\begin{array}{c}
n-1 \\
j
\end{array}\right)(-1)^{j} \int_{0}^{t}\left[\left(1-\mathrm{e}^{-\lambda x}\right)\right]^{n-1} \mathrm{e}^{-\lambda j(t-x)} \mathrm{d} x \\
& =n \lambda\left(1-\mathrm{e}^{-\lambda t}\right)^{n} \sum_{j=0}^{n-1}\left(\begin{array}{c}
n-1 \\
j
\end{array}\right)(-1)^{j} \zeta_{1}(j, n ; t)
\end{aligned}
$$

and, recalling (2.2),

$$
F_{T}^{(n)}(t)=\left(1-\mathrm{e}^{-\lambda t}\right)^{n} \sum_{j=0}^{n}\left(\begin{array}{l}
n \\
j
\end{array}\right)(-1)^{j} \zeta_{0}(j, n ; t) .
$$

Moreover, due to (2.6) and (4.12), we also have

$$
p_{n}(t)=\left(1-\mathrm{e}^{-\lambda t}\right)^{n}-\left(1-\mathrm{e}^{-\lambda t}\right)^{n+1}=\mathrm{e}^{-\lambda t}\left(1-\mathrm{e}^{-\lambda t}\right)^{n},
$$

so that, recalling (2.8), the density of $Y^{+}(t)$ is given, for $0<y<t$, by (4.13).

In conclusion, some plots of the density $\psi^{+}(y ; t)$, obtained in Proposition 4.5, are given in Figure 10 for various choices of $\lambda$ and $t$.

\section{Acknowledgements}

The research of A. Di Crescenzo and B. Martinucci was partially supported by MIUR (PRIN 2008) and by GNCS-INdAM. The research of S. Zacks was partially supported by the Technion, Israel Institute of Technology. The authors thank the anonymous referee for helpful comments that improved the paper.

\section{References}

[1] Abramowitz, M. And Stegun, I. A. (1992). Handbook of Mathematical Functions with Formulas, Graphs, and Mathematical Tables. Dover, New York.

[2] Berg, C. AND Vignat, C. (2008). Linearization coefficients of Bessel polynomials and properties of Student $t$-distributions. Constr. Approx. 27, 15-32. 
[3] Cesarano, C. and Di Crescenzo, A. (2001). Pseudo-Bessel functions in the description of random motions. In Advanced Special Functions and Integration Methods (Melfi, 2000; Proc. Melfi Sch. Adv. Top. Math. Phys. 2), Aracne, Rome, pp. 211-226.

[4] Di Crescenzo, A. (2001). On random motions with velocities alternating at Erlang-distributed random times. Adv. Appl. Prob. 33, 690-701.

[5] Di Crescenzo, A. (2002). Exact transient analysis of a planar random motion with three directions. Stoch. Stoch. Reports 72, 175-189.

[6] Di Crescenzo, A. And Martinucci, B. (2010). A damped telegraph random process with logistic stationary distribution. J. Appl. Prob. 47, 84-96.

[7] Di Crescenzo, A. And Pellerey, F. (2002). On prices' evolutions based on geometric telegrapher's process. Appl. Stoch. Models Business Industry 18, 171-184.

[8] Di Crescenzo, A., Martinucci, B. And Zacks, S. (2011). On the damped geometric telegrapher's process. In Mathematical and Statistical Methods for Actuarial Sciences and Finance, eds C. Perna and M. Sibillo, Springer, pp. 175-182.

[9] Di Matteo, I. And Orsingher, E. (1997). Detailed probabilistic analysis of the integrated three-valued telegraph signal. J. Appl. Prob. 34, 671-684.

[10] Gradshteyn, I. S. and Ryzhik, I. M. (2007). Tables of Integrals, Series, and Products, 7th edn. Academic Press, Amsterdam.

[11] Hansen, E. R. (1975). A Table of Series and Products. Prentice Hall, Englewood Cliffs, NJ.

[12] LaCHal, A. (2006). Cyclic random motions in $\mathbb{R}^{d}$-space with $n$ directions. ESAIM Prob. Statist. 10, $277-316$.

[13] Leorato, S. AND Orsingher, E. (2004). Bose-Einstein-type statistics, order statistics and planar random motions with three directions. Adv. Appl. Prob. 36, 937-970.

[14] Leorato, S., Orsingher, E. And Scavino, M. (2003). An alternating motion with stops and the related planar, cyclic motion with four directions. Adv. Appl. Prob. 35, 1153-1168.

[15] Orsingher, E. (1990). Probability law, flow function, maximum distribution of wave-governed random motions and their connections with Kirchhoff's laws. Stoch. Process. Appl. 34, 49-66.

[16] Orsingher, E. (1990). Random motions governed by third-order equations. Adv. Appl. Prob. 22, $915-928$.

[17] Ricciardi, L. M. (1986). Stochastic population theory: birth and death processes. In Mathematical Ecology (Miramare-Trieste, 1982; Biomath. 17), eds T. G. Hallam and S. A. Levin, Springer, Berlin, pp. 155-190.

[18] Stadje, W. And Zacks, S. (2004). Telegraph processes with random velocities. J. Appl. Prob. 41, $665-678$.

[19] ZACKS, S. (2004). Generalized integrated telegraph processes and the distribution of related stopping times. J. Appl. Prob. 41, 497-507. 